\title{
EVALUATION OF IMPLANT STABILITY AFTER SINUS FLOOR ELEVATION IN POSTERIOR UPPER EDENTULOUS AREA USING TITANIUM MESH WITH SIMULTANEOUS IMPLANT PLACEMENT
}

\author{
Maged Fadl Beshay*, Ramy Ragab El-Beialy ** and Mohammed Galal Beheiri***
}

\begin{abstract}
AIM: The aim of the study was to evaluate the stability of the implants placed simultaneously after graftless sinus floor elevation using titanium mesh and to investigate whether sinus membrane elevation using titanium mesh with simultaneous implant placement (one-stage technique) without grafting materials constitute a valid technique for bone augmentation of sinus floor.
\end{abstract}

Material and methods: The study group comprised 12 patients in whom a total of 17 implants are inserted simultaneously in 12 sinuses. Only blood clots occurring from bleeding due to the surgical procedure filled the secluded compartment space beneath the lifted sinus membrane, where the stable titanium mesh allowing for stable blood clot formation.

Results: All implants achieved adequate primary and secondary stability except one implant showed insufficient second implant stability, six months at the stage of prosthesis fabrication, its secondary stability was inadequate to receive the final restoration and postponed for more and longer follow up (later on, this failed implant was lost and replaced). Overall, measurements taken for all placed implant increased over time, 16 implants achieved adequate secondary stability (ISQ2) and was sufficient for satisfactory loading. It was observed that the larger implant diameter improve the initial implant stability while the length did not had any effect on the initial stability.

Conclusion: Open graftless sinus lift with simultaneous implant placement resulted in bone formation over a period of 6 months and showed high implant survival rates. The treatment time is shorter and decrease the patient discomfort due to reducing the risks of morbidity related to the harvesting of bone grafts.

KEYWORDS: Sinus floor, implant stability, simultaneous implant placement, graftless sinus lift, titanium mesh.

\footnotetext{
* Master Degree of Oral and Maxillofacial Surgery, Faculty of Dentistry, Cairo University. ** Ass. Professor of Oral and Maxillofacial Surgery Faculty of Dentistry Cairo University. *** Professor of Oral and Maxillofacial Surgery, Faculty of Dentistry, Cairo University.
} 


\section{INTRODUCTION}

Insufficient alveolar bone volume is a common limitation in the posterior maxillary region since advanced resorption following premature tooth loss is frequently combined with the pneumatization of the maxillary sinus. ${ }^{(1)}$ Implant therapy is a common way of rehabilitating this area. However, to achieve good primary and secondary implant stability, the treatment is dependent on a certain amount of crestal bone to be successful.(2) Therefore, various maxillary sinus floor augmentation techniques have been used for reconstruction of the posterior maxilla in conjunction with simultaneous or delayed placement of the dental implants..$^{(3,4)}$

There has been a considerable clinical controversy about the role of the graft material in the sinus lift procedure. In addition, there is inconclusive evidence to prove the need for grafting material in direct sinus lift procedures eventually, the idea of a graft-free augmentation of the maxillary sinus has evolved. ${ }^{(5)}$ A non-grafted sinus augmentation procedure is thus vital to maintain stable blood clot formation during the postoperative healing stage, and additional techniques may be required to allow the membrane to maintain a lifted position in the long term. ${ }^{(6)}$

Therefore, simultaneous implant placement is required to maintain blood clot in the new created compartment under the elevated sinus membrane when bone materials are not used.(7) The one-step lateral antrostomy is used for the residual bone height of 4 to $6 \mathrm{~mm}$ for achieving primary implant stabilization, and the less invasive osteotome technique is recommended when more than $6 \mathrm{~mm}$ of residual bone height is available. ${ }^{(8,9)}$ Adequate bone is required to achieve sufficient primary stability in case of sinus floor elevation with simultaneous implant placement. It is thought that when the minimum residual bone height is not less than $4 \mathrm{~mm}$, there should be a sufficient layer of cortical bone in the sinus floor to achieve the initial stability. ${ }^{(10)}$

\section{MATERIALS AND METHODS}

The current study was carried out on 12 maxillary sinuses, where all participants seeking fixed dental prostheses for their posterior maxilla with a limited bone height below the maxillary sinus floor secondary to sinus pneumatization. They were selected from the outpatient clinic of Oral and Maxillofacial Surgery Department, Faculty of Dentistry, Cairo University.

\section{Study design:}

Description of trial design: Clinical trial.

Type of trial: Single blind /statistics.

\section{Eligibility criteria:}

The patients were selected according to the following criteria:

\section{- Inclusion criteria:}

(1) The residual alveolar bone height beneath the maxillary sinus floor should be not less than $4 \mathrm{~mm}$. $(\geq 4 \mathrm{~mm} \leq 6 \mathrm{~mm}$.)

(2) Age of the patients more than 18 years.

(3) Patients are systemically and medically free from any immunological diseases that could affect normal bone healing.

(4) Sufficient inter-arch space for future prosthesis.

\section{- Exclusion criteria:}

(1) Patient with history of systemic illness.

(2) An active current infection or history of persistent sinus infection.

(3) The presence of uncontrolled diabetes.

(4) History of chemotherapy or radiotherapy to the head and neck.

\section{Pre-operative assessment}

As regarding the medical history, all patients were free from any systemic diseases. Comprehensive 
intraoral examination and dental history carefully taken was performed. A computed tomography (CT) scan was taken from a patient to obtain dicom format images. MSCT will confirm the exact RBR; in addition, to asses presence of any sinus pathology, condition or septa and detection of the sites which will receive the dental implants.

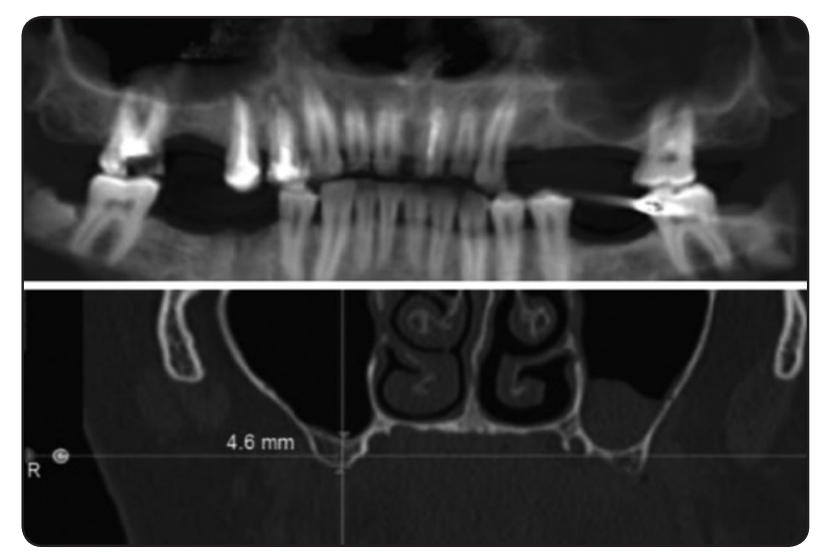

Fig. (1): Reformatted panoramic view, showing moderate pneumatization (right side) while (left side) showing sever pneumatization and Preoperative MSCT coronal view showing the measurement of residual bone height (right maxillary sinus).

\section{Surgical procedures}

All the surgical procedures were performed under local infiltration anesthesia administrated in the buccal vestibules and the palatal mucosa opposite the site of surgery.

A full-thickness mid-crestal incision slightly palatal to the crest of the ridge to be sure that the implant will not be in the line of incision or in the way of suture. Anterior and posterior vertical releasing incisions were made of sufficient length and in a slightly flaring direction to provide both good basal blood supply for the created flap and sufficient clinical access and vision.

The mucoperiosteal flap was reflected to fully expose the alveolar ridge and the lateral wall of the maxillary sinus. Access to the maxillary sinus is obtained by drilling a bony window in the lateral sinus wall using a diamond round surgical bur $8 \mathrm{~mm}$ diameter, under constant irrigation with sterile saline solution to create an oval or rectangularshaped outline in the lateral wall of the maxillary sinus. Upon thinning of the bone, a bluish hue of the membrane should be noted. Special elevators (freers) are used to carefully to release the lateral bony window with the underlying membrane attached to it from the periphery of the osteotomy outline to get a cleavage plane.

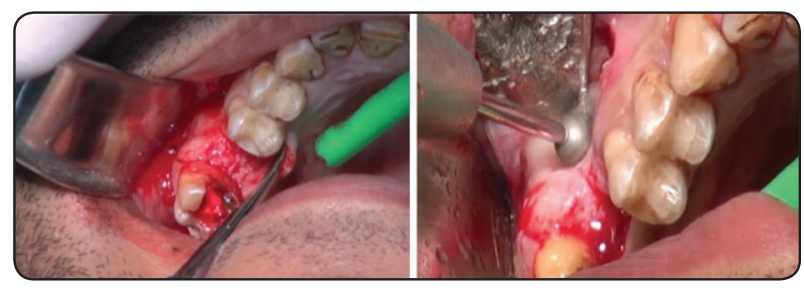

Fig. (2): Showing surgical flap and showing diamond round surgical bur $8 \mathrm{~mm}$ diameter performing Access to the maxillary sinus under copious coolant.

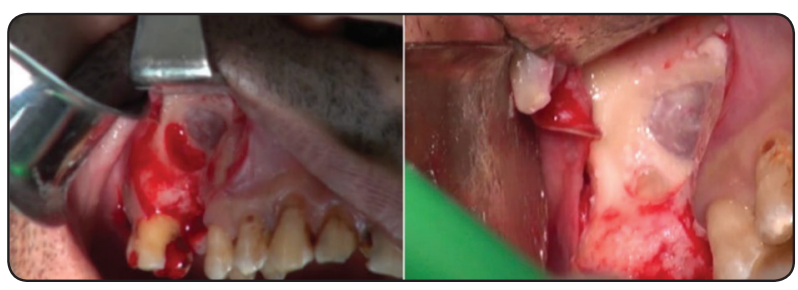

Fig. (3): Showing delineation of the outline of the rectangular osteotomey without perforating the antral membrane. (Bluish hue of the membrane)

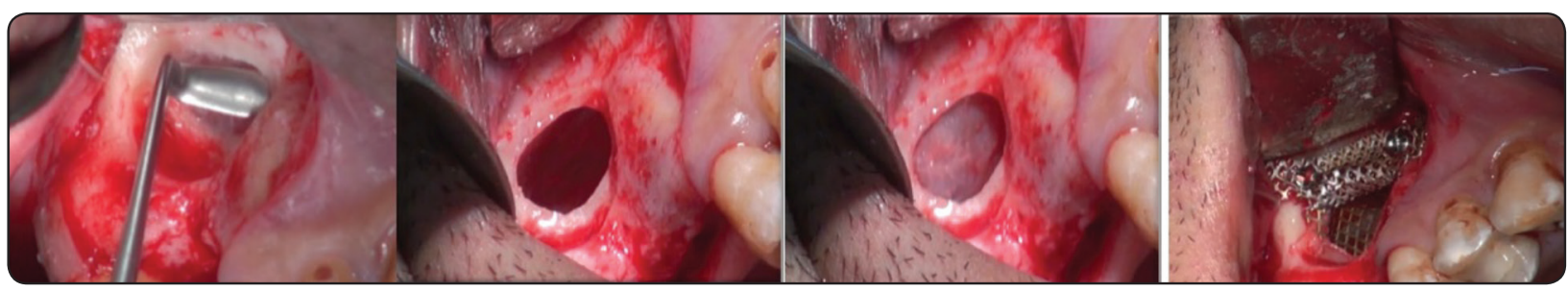

Fig. (4): Showing application of freer to elevate sinus membrane and Showing fixation of the titanium mesh to the lateral wall of the sinus with micro screws. 
- $0.1 \mathrm{~mm}$ thickness micro titanium mesh fixed to the lateral wall of the sinus above the superior osteotomy with two $1.5 \mathrm{~mm}$ micro screws. Mesh it will ultimately serve as the new sinus floor. Implants were placed according to the standard manufacturer systems protocol and a performed by dentium surgical kit. Implant stability quotient (ISQ) was recorded at the time of implant placement (baseline-ISQ1) and 6 months after placement (ISQ2), at the time of implant loading.

RFA measurements were carried out in 4 perpendicular directions, mesio-distal [MD], buccolingual [BL], disto-mesial [DM] and linguo-buccal

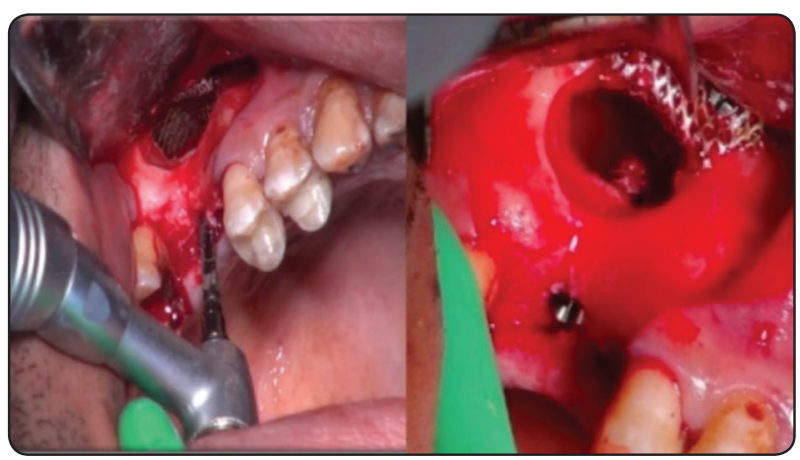

Fig. (5): Showing drilling for implant placement and the titanium mesh uplifted the sinus membrane.

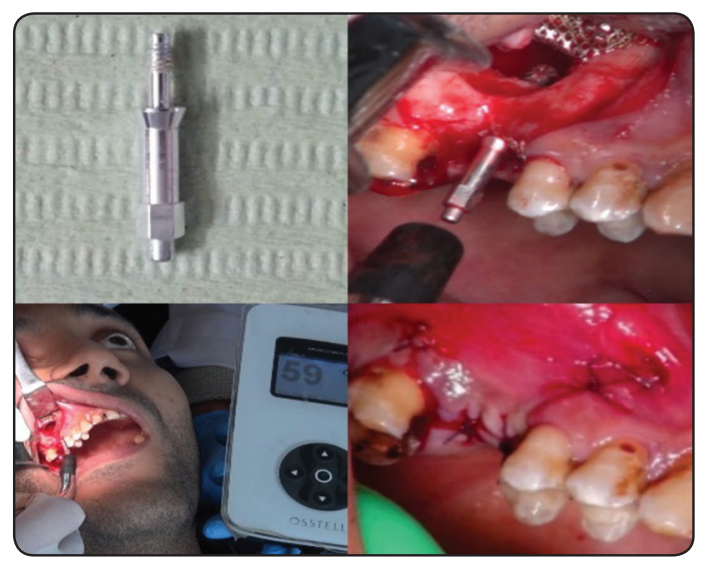

Fig. (6): Smart peg attached to the implant (ISQ1 measurement) and wound closure by simple interrupted sutures.
[LB], where the average mean value was calculated, (a mean general value for the 4 directions were calculated). The flap was repositioned and wound closure by simple interrupted sutures using 3-0 resorbable (3-0 resorbable vicryl) that were removed after 10 days.

Two days post-surgerically, patients were recalled for wound inspection for any signs of bleeding, infection, hematoma or wound dehiscence. Radiographic examinations (MSCT) were performed immediately postoperatively one day and 6 months after the surgery using the same CT machine and the same exposure parameters.

- Linear measurements were performed to measure and evaluate the existing maxillary alveolar bone height before and at 6 months after surgery and the gain in bone height was measured by comparing the preoperative and final CT scans using special software programs, White Fox software (version 4.0 2014, Paris, France).

- Density readings were recorded as gray scale values at the same area measured for height. The native bone density around the implant (measurements were done at the mesial, distal, buccal and palatal aspect of each implant and their average was taken) evaluated in reference to the density of the newly formed bone at the area inferior to the titanium mesh, the density of bone was measured in Hounsfield Units.

\section{Secondary implant stability measurement and prosthetic stage}

After the completion of the healing period (6 months post- operatively) right before the healing abutment connection, patients were recalled for measurement of biological or secondary implant stability. 


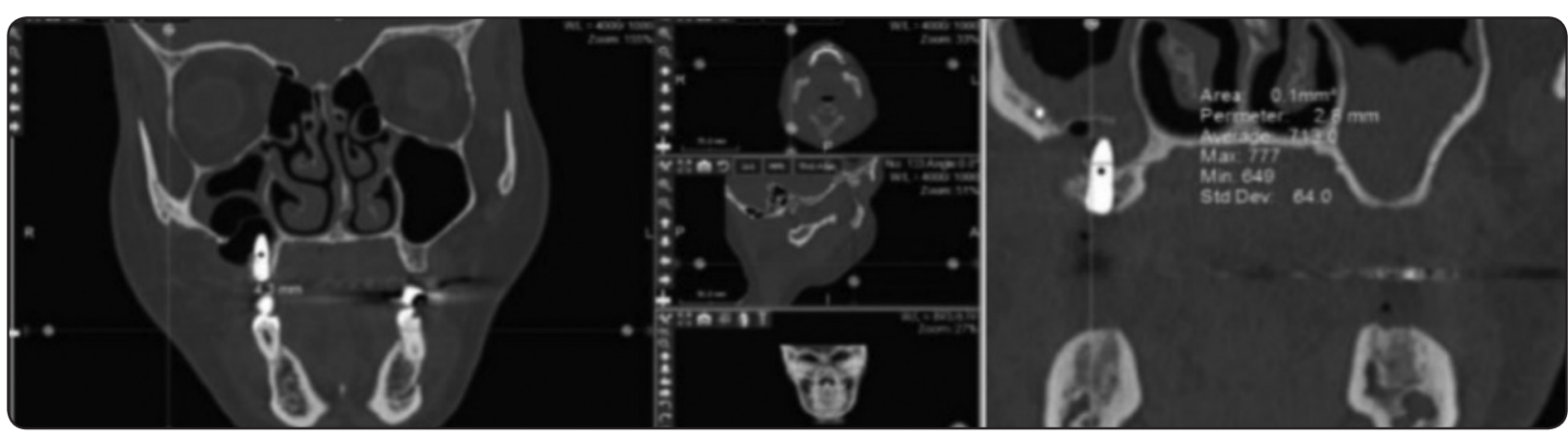

Fig. (7): Immediate postoperative coronal view MCST showing measurement of RBH around implant and showing measurement for the native bone density .

\section{RESULTS}

- All patients included in the study were examined preoperatively, immediately after the surgical procedures and 6 months later as a follow up. None of the patients displayed any sign of purulent discharge or fever throughout our study period. Only one patient showed delayed wound healing and 2 weeks later it healed completely. 2 patients developed sinusitis subsequently which was managed early by antibiotics and nasal decongestant.

\section{Clinical assessment}

- All implants were placed properly and there was a space between the apical end of the implant and the titanium mesh where the space between the residual bone and the titanium mesh is called the sub-sinus space (the created compartment).

- At six month post operatively, at the stage of prosthetic construction, the soft tissue surrounding the implants looked healthy without signs of inflammation. All the implants were surgically exposed and healing abutments were installed followed by porcelain fused to metal crown and bridges.

\section{Six months postoperatively}

Qualitative assessment of MSCT radiographs showed the followings:

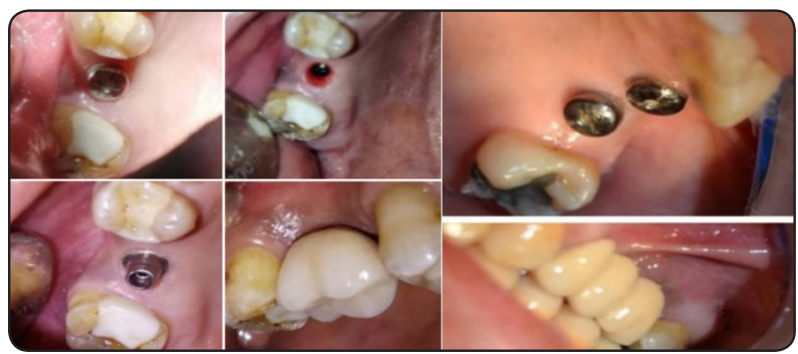

Fig. (8): a. Healing cap for different cases, b. soft tissue around implant, c. abutment and d. porcelain crown in different cases.

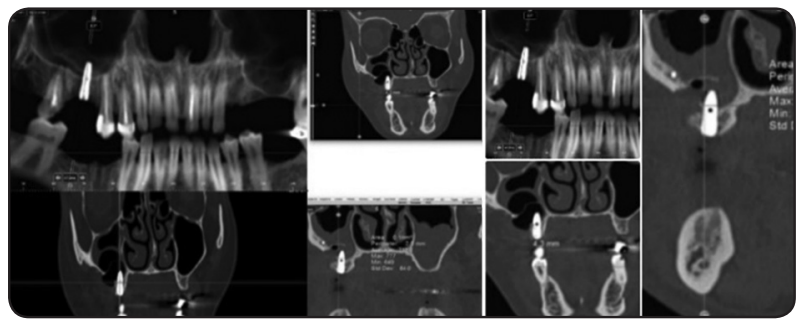

Fig. (9): Immediately postoperative radiographic panoramic \& MSCT views showing proper properly placed Sshaped mesh, implant and the created sub-sinus space.

New bone regenerate in all cases beneath the titanium mesh, the newly formed bone was less dens than the adjacent native alveolar bone. The regenerated bone appeared as a convex dome-shape radiopacity with a round margins under the elevated membrane (titanium mesh) (Fig. 10).

$\leftarrow$ Bone Density: Comparison between bone density measurements and its percentage showed statistically significant higher mean bone density in the native bone more than the newly formed bone at six months postoperatively. 


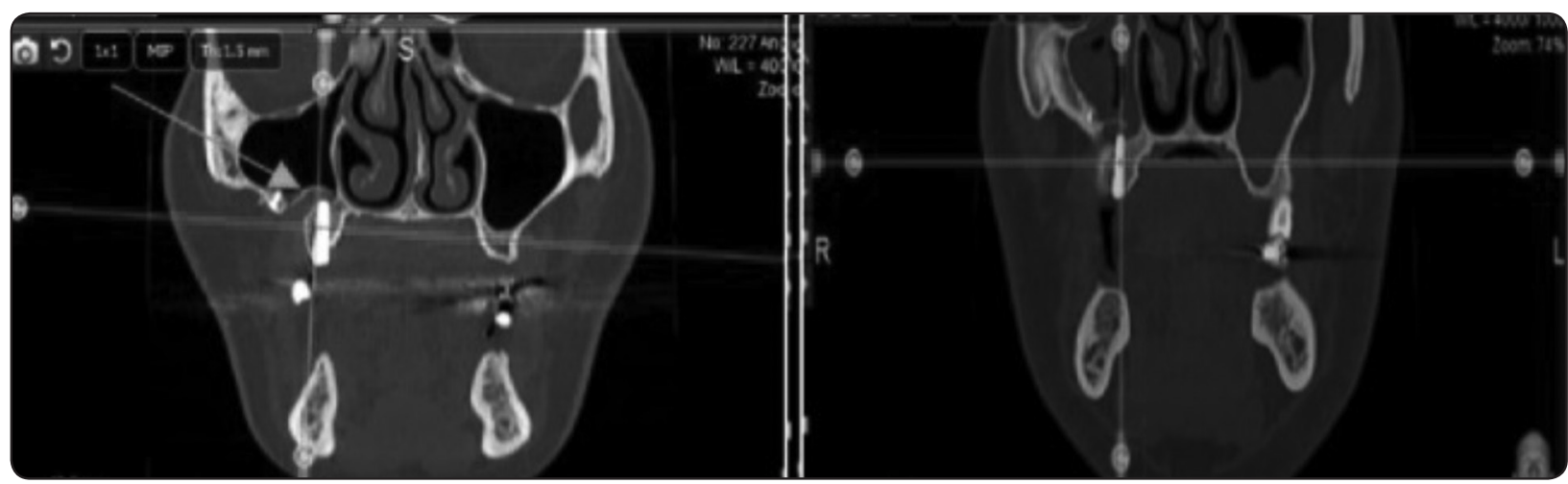

Fig. (10): Postoperative follow up 6 months coronal MSCT showing dome shaped bone formation beneath the properly placed mesh (blue arrow).

$\leftarrow$ The results showed that there was a highly statistically significant difference between immediate and after 6months according to alveolar buccal bone, alveolar palatal bone, alveolar mesial bone, alveolar distal bone and mean of native bone density. (Table 1).

\section{Implant monitoring}

All implants achieved adequate primary stability even in a thin residual bone height. Overall, measurements taken for all placed implant increased over time, 16 out of 17 implants achieved adequate secondary stability (ISQ2) and was sufficient for satisfactory loading.

The implant stability six months (secondary stability) after placement is significantly higher than the stability recorded at the time of implant placement (primary stability). There was a significant difference between primary stability values (ISQ1) and secondary stability values (ISQ2). This could be explained by the occurrence of osseointegration (Fig. 11 and 12) (Table 2).

\section{Correlation between primary stability and native bone density (Bone density and implant stability)}

The results of the present study showed that the initial stability increased according to the increase in the native bone density, resulting in a strong positive correlation. In other words, the initial stability was shown to be highly dependent on the residual bone density.

TABLE (1) Comparison between Immediate postoperative (Native bone density) and 6 months follow up (Newly formed bone density) of the study group.

\begin{tabular}{|c|c|c|c|c|c|}
\hline Bone density & $\begin{array}{l}\text { Immediate postoperative } \\
\text { (Native bone density) } \mathrm{HU}\end{array}$ & $\begin{array}{l}6 \text { months follow up (Newly } \\
\text { formed bone density) HU }\end{array}$ & Mean Diff. & $\begin{array}{c}\text { Paired } \\
\text { t-test }\end{array}$ & p-value \\
\hline Alveolar buccal bone & $236-653.5[497.09 \pm 126.34]$ & $220-557[333.29 \pm 95.75]$ & -163.80 & 8.642 & $<0.001 * *$ \\
\hline Alveolar palatal bone & $277.5-610.5[459.26 \pm 97.00]$ & $185.5-524[305.71 \pm 87.63]$ & -153.55 & 11.337 & $<0.001 * *$ \\
\hline Alveolar mesial bone & $292.5-575.5[438.56 \pm 85.86]$ & $203-475.5[287.50 \pm 78.34]$ & -151.06 & 12.195 & $<0.001 * *$ \\
\hline Alveolar distal bone & $253.5-591[429.94 \pm 96.27]$ & $188-505.5[282.71 \pm 80.95]$ & -147.23 & 11.284 & $<0.001 * *$ \\
\hline Mean of native bone density & $267.63-600[456.22 \pm 97.35]$ & $206.25-515.5[302.30 \pm 83.72]$ & -153.92 & 11.964 & $<0.001 * *$ \\
\hline
\end{tabular}


TABLE (2) Comparison between immediate and after 6 months according implant stability.

\begin{tabular}{|l|c|c|c|c|}
\hline \multicolumn{1}{|c|}{ Implant stability } & Range $[$ Mean \pm SD] & Mean Diff. & Paired t-test & p-value \\
\cline { 1 - 3 } Immediate & $53-67.75[61.75 \pm 3.98]$ & \multirow{2}{*}{10.6} & 28.225 & $<0.001 * *$ \\
\cline { 1 - 4 } After 6months & $60.75-79.5[72.35 \pm 4.59]$ & & & \\
\hline
\end{tabular}

t-Paired Sample t-test; **p-value $<0.001 \mathrm{HS}$

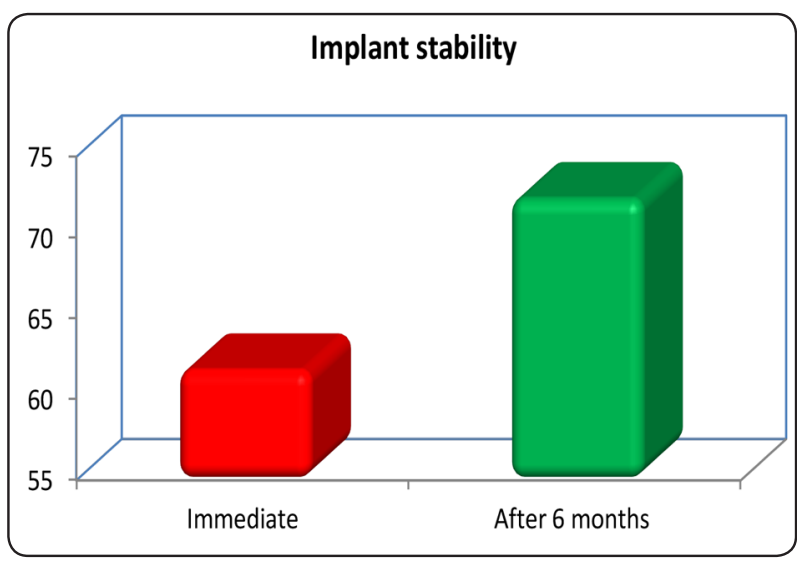

Fig. (11): Bar chart between immediate and after 6 months according implant stability.

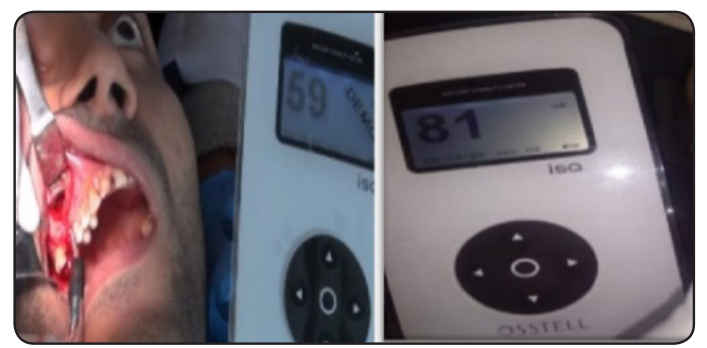

Fig. (12): Showing primary and secondary implant stability (ISQ2) measurement by using osstell device ( the same case after 6 months)

\section{DISCUSSION}

Rehabilitation of atrophied edentulous posterior maxillary region with implants represents a challenge especially in case of inadequate bone volume caused by pneumatization of the maxillary sinus together with resorption of alveolar ridge due to long term edentulism and biological aging. ${ }^{(11)}$ Maxillary sinus floor elevation offers one of the most common pre-prosthetic procedures to solve this problem. ${ }^{(12)}$
The current study procedure describes a recent trend in maxillary sinus augmentation method using the patients' own blood from the surgical wound of the lateral window osteotomy, without any bone graft material, for the implant treatment for rehabilitation of the atrophied posterior maxillary region, where blood clot formation serves as autogenous graft filler material for bone regeneration during graftless maxillary sinus lifting.

The present study was designed to evaluate clinically the stability for the placed implant simultaneously inserted in non-grafted or graftless sinus floor elevation by using titanium mesh in addition to assess the validity on one stage surgical stage or technique.

In the present study, using titanium mesh as a space maintainer device provides an effective mechanical method to preserve the newly created subsinus space after Schneiderian membrane elevation, allowing for stable blood clot formation and filling is not absolutely necessary because the natural blood clot inside the subsinus chamber is enough for bone healing. ${ }^{(13)}$

Ikumi et al., revealed that residual bone amount is an important factor in obtaining proper implant stability and the lack of alveolar bone is a limiting factor for implantation, our own findings coincide with this previous observation. ${ }^{(14)}$ On the contrary, previous clinical studies by Urban et al., ${ }^{(15)}$ and Moeintaghavi et al., ${ }^{(16)}$ showed that there was no relationship between ISQ values and bone height (residual vs. augmented or newly bone gain height), also determined an equal success with minimal and moderate residual bone heights. 
The results of the present study showed that, primary implant stability (ISQ1) values were positively significant correlated to native residual bone densities, a high value of primary stability will be achieved, in case of higher bone density. The obtained clinical and radiographic results proved that the initial implant stability is mostly determined by the bone density at the surgical site.

In the present study, the differences in the primary stability values between the different cases are due to the relative differences of the residual bone densities in between the cases.

The present study correlates between the implant stability (ISQ) with the density (Hu) of the residual bone. Regarding the relationship between bone density in $\mathrm{Hu}$ and primary implant stability, a significant statistical relationship was obtained between different native bone qualities and the implant stability, measured with ISQ values, was found greater ISQ values obtained with the increased or high native bone density $(\mathrm{Hu})$, this finding is in agreement with previous clinical studies reported by the Huang et al., ${ }^{(17)}$ and Shahlaie et al., ${ }^{(18)}$ demonstrated that there is a positive relation between the bone density and the implant stability.

In the current study, implant stability quotient (ISQ) was measured using osstell device at the time of implant placement (immediate implant stability ISQ1) and 6 months after implant placement (secondary implant stability ISQ2) at the stage of the implant loading by the final restoration. Resonance frequency analysis was chosen as a non-invasive, reliable and reproducible method to assess primary stabilities and monitor them over time.

The present study showed that after the healing period, there was a significant difference between the primary stability values (ISQ1) and secondary stability values (ISQ2) and this could be explained by the occurrence of osseointegration and the newly formed bone occurred around the implants. Changes in implant stability that occurred, have been attributed to a delay in bone healing and that explain the increase in the implant stability in ISQ2 values.

In the present study bone formation was evident around the implants. In all cases, the new bone formation was notable, with good continuity with the native sinus floor. The newly formed bone on the maxillary sinus floor was clearly discernible around and on the apical side of the dental implants and beneath the titanium mesh, this result is in accordance with the previous clinical studies in humans. ${ }^{(10,19,20)}$

The current study showed that, there are two important key roles in sinus bone reformation, a first key role for this bone formation lies in Schneiderian membrane and the bone gain is not fully cleared process. In graftless sinus elevation technique, blood clot after sinus tenting acts as an autologous osteogenic graft material, to which osteoprogenitors can migrate, differentiate, and regenerate bone. ${ }^{(21,22)}$

The second role for bone reformation in the sinus is not grafting materials, but space making under the elevated sinus membrane. Therefore, simultaneous implant placement is preferred to maintain blood clot in the new compartment under the elevated sinus membrane when bone materials are not used. $(12,23)$

The outcomes of the present study revealed that survival rate increased steadily with increasing ISQ reading, so securing the implant primary (mechanical) stability is positively associated with a successful implant integration and long-term successful clinical outcome, therefore, it is essential to assess the initial stability to ensure a successful osseointegration and this is in agreement with other many studies. ${ }^{(24,25,26)}$

The present study showed that the amount of residual bone density and height significantly influenced the implant survival after graft-free sinus floor elevation, but in conclusion, the implant 
survival rate can be affected by many different factors like surgical technique, implant diameter achieving sufficient primary stability and $\mathrm{RBH}$ cannot be singled out as the only factor for implant survival.

The present study highlights the importance of achieving a sufficient primary stability which represents the basic requirement to guarantee a correct healing of the implant for successful implant integration especially in performing the one stage technique graftless sinus lift and immediate implant placement by using wide diameter and undersizing the implant bed preparation to improve the initial implant stability.

Performing one stage technique and simultaneous implant placement with graft free sinus floor elevation in atrophic posterior maxilla provides clinicians with a more conservative option of the treatment and help to minimize treatment period, cost and trauma.

The performed graftless sinus lift with immediate implant placement (one stage technique) offers the following advantages: (1) there is no need for a donor site so, patient's morbidity is very low because the harvesting of autogenous bone is not required, (2) less cost, (3) There is less contamination associated with this procedure as there is no need to get an allograft of animal origin or an alloplastic material,(4) in case of membrane perforation, there is no risk of penetration of the grafting material in the sinus, (5) minimize patient discomfort by reducing surgeries's numbers and shortening treatment time.

\section{CONCLUSION}

From the results of the current study, it could be concluded that:

1) Simultaneous open sinus lift \&implant placement is a good reliable technique for increasing the bone volume and show high implant survival rates.
2) Graftless sinus lift by using titanium mesh to maintain a stable blood clot is a valid and reliable method \& using graft is not essential anymore for sinus lifting procedures.

3) The technique is considered to be cost-effective as no graft material is needed, less time-consuming, fewer patient surgeries, lower infection risk, as it does not involve a biomaterial, and reducing the risks for morbidity related to harvesting of bone grafts.

4) Resonance frequency analysis is a non-invasive, good and easy method used to determine implant stability and to predict the implant healing throughout the follow up period, in addition to predict the success or failure of the implant.

\section{REFERENCES}

1. Asai S, Shimizu Y, Ooya K. Maxillary sinus augmentation model in rabbits: effect of occluded nasal ostium on new bone formation. Clin Oral Implants Res. 2002;13(4):405-409.

2. Corbella S, Taschieri S, Del Fabbro M. Long-term outcomes for the treatment of atrophic posterior maxilla: a systematic review of literature. Clin Implant Dent Relat Res. 2015;17(1): 120-132.

3. Hatano N, Shimizu Y, Ooya K. A clinical long-term radiographic evaluation of graft height changes after maxillary sinus floor augmentation with a 2:1 autogenous bone/xenograft mixture and simultaneous placement of dental implants. Clin Oral Implants Res. 2004;15(3):339-345.

4. Hallman M, Sennerby L, Zetterqvist L, Lundgren S. A 3-year prospective follow-up study of implant-supported fixed prostheses in patients subjected to maxillary sinus floor augmentation with a 80:20 mixture of deproteinized bovine bone and autogenous bone Clinical, radiographic and resonance frequency analysis. Int J Oral Maxillofac Surg. 2005;34(3):273-280.

5. Badr M, Oliver R, Pemberton P, Coulthard P. Platelet-Rich Plasma in Grafted Maxillae: Growth Factor Quantification and Dynamic Histomorphometric Evaluation. Implant Dent. 2016;25(4):492-498.

6. Chen TW, Chang HS, Leung KW, Lai YL, Kao SY. Implant placement immediately after the lateral approach of the trap door window procedure to create a maxillary 
sinus lift without bone grafting: a 2-year retrospective evaluation of 47 implants in 33 patients. J Oral Maxillofac Surg. 2007;65(11):2324-2328.

7. Li X, Rong Q, Chen S-L. The innate osteogenic potential of the canine maxillary sinus membrane: An in vitro and in vivo study. J Biomater Tissue Eng. 2015;5(6):445-451.

8. Zitzmann NU, Schärer P. Sinus elevation procedures in the resorbed posterior maxilla. Comparison of the crestal and lateral approaches. Oral Surg Oral Med Oral Pathol Oral Radiol Endod. 1998;85(1):8-17.

9. Summers RB. A new concept in maxillary implant surgery: the osteotome technique. Compendium. 1994;15(2):152-162.

10. Lundgren S, Cricchio G, Palma VC, Salata LA, Sennerby L. Sinus membrane elevation and simultaneous insertion of dental implants: a new surgical technique in maxillary sinus floor augmentation. Periodontol 2000. 2008;47(1):193-205.

11. Scarano A, Degidi M, Iezzi G, et al. Maxillary sinus augmentation with different biomaterials: a comparative histologic and histomorphometric study in man. Implant Dent. 2006;15(2):197-207.

12. Pandit A, Chopra S. Maxillary sinus floor elevation: review of anatomy and lateral sinus lift technique. IOSR J Dent Med Sci. 2016;1:29-33.

13. Kaneko T, Masuda I, Horie N, Shimoyama T. New bone formation in nongrafted sinus lifting with space-maintaining management: a novel technique using a titanium bone fixation device. J Oral Maxillofac Surg. 2012;70(3):e217-e224.

14. Ikumi N, Tsutsumi S. Assessment of correlation between computerized tomography values of the bone and cutting torque values at implant placement: a clinical study. Int J Oral Maxillofac Implants. 2005;20(2):253-260.

15. Urban IA, Lozada JL. A prospective study of implants placed in augmented sinuses with minimal and moderate residual crestal bone: results after 1 to 5 years. Int J Oral Maxillofac Implants. 2010;25(6):1203-1212.

16. Moeintaghavi A, Ghanbari H, Radvar M, Zare R, Sargolzaei N, Nicknam H. Resonance frequency analysis of implant stability in augmented and non-augmented sinus sites. J Dent Mater Tech. 2016;5(1):43-48.

17. Huang HM, Lee SY, Yeh CY, Lin CT. Resonance frequency assessment of dental implant stability with various bone qualities: a numerical approach. Clin Oral Implants Res. 2002;13(1):65-74.

18. Shahlaie M, Gantes B, Schulz E, Riggs M, Crigger M. Bone density assessments of dental implant sites: 1. Quantitative computed tomography. Int J Oral Maxillofac Implants. 2003;18(2):224-231.

19. Thor A, Sennerby L, Hirsch JM, Rasmusson L. Bone formation at the maxillary sinus floor following simultaneous elevation of the mucosal lining and implant installation without graft material: an evaluation of 20 patients treated with 44 Astra Tech implants [published correction appears in J Oral Maxillofac Surg. 2008 Oct;66(10):2195-6]. J Oral Maxillofac Surg. 2007;65(7 Suppl 1):64-72.

20. Balleri P, Veltri M, Nuti N, Ferrari M. Implant placement in combination with sinus membrane elevation without biomaterials: a 1-year study on 15 patients [published correction appears in Clin Implant Dent Relat Res. 2013 May 6;15(3):470. Piero, Balleri [corrected to Balleri, Piero]; Mario, Veltri [corrected to Veltri, Mario]; Niccolò, Nuti [corrected to Nuti, Niccolò]; Marco, Ferrari [corrected to Ferrari, Marco]]. Clin Implant Dent Relat Res. 2012;14(5):682-689.

21. Atef M, Hakam MM, ElFaramawey MI, Abou-ElFetouh A, Ekram M. Nongrafted sinus floor elevation with a spacemaintaining titanium mesh: case-series study on four patients. Clin Implant Dent Relat Res. 2014;16(6):893-903.

22. Starch-Jensen T, Jensen JD. Maxillary Sinus Floor Augmentation: a Review of Selected Treatment Modalities. J Oral Maxillofac Res. 2017;8(3):e3. Published 2017 Sep 30.

23. Bell GW, Joshi BB, Macleod RI. Maxillary sinus disease: diagnosis and treatment. Br Dent J. 2011;210(3):113-118.

24. Javed F, Ahmed HB, Crespi R, Romanos GE. Role of primary stability for successful osseointegration of dental implants: Factors of influence and evaluation. Interv Med Appl Sci. 2013;5(4):162-167.

25. Turkyilmaz I, Aksoy U, McGlumphy EA. Two alternative surgical techniques for enhancing primary implant stability in the posterior maxilla: a clinical study including bone density, insertion torque, and resonance frequency analysis data. Clin Implant Dent Relat Res. 2008;10(4):231-237.

26. Rodrigo D, Aracil L, Martin C, Sanz M. Diagnosis of implant stability and its impact on implant survival: a prospective case series study. Clin Oral Implants Res. 2010;21(3):255-261. 